\title{
Peripheral nerve function in patients with bronchial carcinoma. Comparison with matched controls and effects of treatment
}

\author{
J A R LENMAN, A M FLEMING, M A R OBERTSON, \\ R J ABBOTT, M D CLEE, I F FERGUSON, AND D S WRIGHT \\ From the Section of Neurology, Department of Medicine, Ninewells Hospital and Medical School, \\ Dundee, and the Department of Respiratory Disease, Kings Cross Hospital, Dundee
}

S U M MARY Clinical examination of 80 patients with bronchial carcinoma showed minor neurological abnormalities but in only a few cases were these considered to be due to neuromyopathy. Spontaneous activity in the EMG was shown in $35 \%$, consistent with a mild degree of partial denervation; when 50 of the patients were matched with 50 controls the patients showed a small but significant impairment of nerve conduction velocity in comparison with the controls. These findings accord with subclinical neuropathy in a high proportion of patients with bronchial carcinoma consistent with primary axonal change. Thirty patients participated in a prospective study of the effects of treatment. Of these nine were reassessed following surgery or radiotherapy. Although there was a trend towards improvement in sensory conduction there was no consistent change in the electromyographic findings.

Although the occurrence of non-metastatic changes in the nervous system in association with carcinoma had been recognised previously, the first definitive description of such a syndrome was in 1948 when Denny-Brown ${ }^{1}$ described two cases of sensory neuropathy in patients with carcinoma of the lung. Many disorders of nerve and muscle as well as the central nervous system have since been recognised and Brain and Henson have classified them under the general term of carcinomatus neuromyopathy. ${ }^{2}$ Although these can occur in association with cancer in many different sites, the highest incidence is in association with bronchial carcinoma and in one study of 316 patients with this condition $14 \cdot 2 \%$ had clinical evidence of neuropathy. ${ }^{3}$ Electrophysiological studies have shown that the incidence of subclinical neurological or myopathic abnormalities in cancer patients may exceed $50 \% .^{4-7}$

Information regarding the natural history of neuropathy and in particular the effects of treat-

\footnotetext{
Address for reprint requests: Dr JAR Lenman, Department of Medicine, Ninewells Hospital and Medical School, Dundee DD1 9SY, Scotland.

Accepted 12 October 1980
}

ment of the underlying carcinoma remains incomplete. Brain and Henson $^{2}$ described two patients in whom neuropathy occurred for the first time eight years after reseciion of the bronchial carcinoma. Croft and Wilkinson ${ }^{8}$ examined 250 patients with bronchial carcinoma of whom 35 had been treated either surgically or with radiotherapy. The authors considered that the incidence of neuropathy was commoner in the treated than in the untreated patients. In a later study it was noted that symptoms might precede evidence of malignant disease ${ }^{9}$ by several months and it was observed that neurological improvement would not take place where the neuropathic changes were confined to the central nervous system, but might sometimes occur in patients with peripheral nerve involvement.

In the present study we have examined 50 patients with a clinical diagnosis of bronchial carcinoma and carried out electrophysiological studies to compare the findings with a group of age-matched controls. In addition we have carried out a prospective study on a further group of $\mathbf{3 0}$ patients with bronchial carcinoma to try and assess the effects of treatment. 


\section{Methods}

In the first part of the study 50 patients with bronchial carcinoma were examined clinically and electromyographically, and measurements of nerve conduction velocity were compared with those in 50 age matched controls. In the second part, a group of 30 patients with bronchial carcinoma was studied and the examination was repeated in those patients who were available six months after treatment. In this group the electromyographic findings were compared with those in a second group of control subjects.

\section{A Comparison of 50 patients with bronchial carcinoma} and 50 controls

The patients were referred from the regional chest hospital and the only selection in the referral of patients was that the patients were fit enough to come to the laboratory and undergo examination. The controls were either members of hospital staff or patients in a medical ward without symptoms of carcinoma or neurological disease who gave their informed consent. They were of the same sex (49 male, 1 female) and race as the patients and the same age to within 5 years. The mean age of the patients was 61.9 years, of the controls 60.7 years. Each patient and control was examined clinically according to a standardised protocol and at the same time blood was taken from each patient for haematological and biochemical examination including blood sugar and estimation of serum creatine kinase and aldolase.

Each patient and control had an electroencephalogram comprising a routine record with hyperventilation and photic stimulation. Fastest motor conduction velocity was measured in patients and controls in the right and left median, ulnar and common peroneal nerves using surface electrodes according to procedures described previously. ${ }^{10}$ Sensory conduction velocity was measured in the median and ulnar nerves. In addition the peak to peak amplitude of evoked responses in nerve and muscle and the duration of the evoked response in muscle were measured from photographed oscilloscope traces. Measurements were made in warm surroundings and the extremities were warmed if skin temperatures locally were less than $30^{\circ} \mathrm{C}$. Mean temperatures for upper and lower extremities were $31.1^{\circ} \mathrm{C}$, SD 1.19 for patients and $31.2^{\circ} \mathrm{C}$, SD 1.30 for controls.

Electromyography was carried out using a concentric electrode in the 50 patients but not in the controls. In each patient the right and left deltoid and quadriceps muscles were studied and in 36 the examination included the tibialis anterior muscles. Nerve conduction was measured using a Tektronix 564 storage oscilloscope and 122 preamplifier. For electromyography a Stanley Cox 5D electromyograph was used.

B Prospective study of bronchial carcinoma patients before and after treatment

Initially 32 patients (20 male and 12 female) were studied but two were excluded because the diagnosis was changed following thoracotomy. The patients were selected following bronchoscopy and all but two were considered to have a surgically treatable tumour. In 27 of the 30 patients the bronchial carcinoma was proven by histology; in the remaining three it was strongly suspected on clinical grounds. Nine patients who had been treated for bronchial carcinoma subsequently returned for further study after six months. The remainder either had died or were otherwise unable to return for reassessment. Twenty control patients who were free of neurological or malignant disease also were studied. These were patients either from a chest diseases or from a urology ward who gave informed consent for the procedure. The mean age of the patients with bronchial carcinoma was 62.0 years, of the controls $57 \cdot 8$ years.

All patients were interviewed and examined clinically prior to each EMG study. Electromyography using a concentric electrode was performed in 31 patients (29 with carcinoma) and also in the controls. In every patient the right deltoid, first dorsal interosseus, quadriceps and tibialis anterior muscle was studied along five separate tracks of electrode insertion. In addition in 24 of the patients and 18 of the controls the right deltoid muscle was explored and 20 motor unit potentials were displayed by means of a delay line and photographed on at least three occasions at sweep velocities of 2 and $5 \mathrm{~ms} / \mathrm{cm}$. These potentials were examined and changes in phase of greater than $50 \mu \mathrm{V}$ were counted. Nerve conduction measurements were performed in all the patients with bronchial carcinoma using the same methods and the same nerves as in the 50 patients and 50 controls already described and using the same methods of temperature control. In this study, however, all measurements were made using a Medelec MS6 recording system.

\section{Results}

A Comparison of 50 patients with bronchial carcinoma and 50 controls.

Clinical findings None of the patients presented with a primary neurological problem, but some neurological abnormality (often of a minor nature) was found in 31 . These included hemiparesis in one patient, hoarseness of voice in seven patients and an extensor plantar response in two. Three patients had some local wasting and other signs included absent ankle jerks and depressed vibration sense in the lower limbs. In four patients a diagnosis of cerebral metastasis was made later. Seven of the controls also were found to have minor neurological signs which included absent ankle jerks, depressed vibration sensc, some unsteadiness of gait and nystagmus.

EEG findings The electroencephalogram was 
within normal limits in 33 of the patients with bronchial carcinoma as compared with 38 of the control series. The control group however was considered not to be entirely satisfactory in terms of EEG normality in view of the high proportion of cardiac and respiratory cases in a hospital population of the age group under study. The 17 abnormal records from the patients with bronchial carcinoma included four with borderline and 13 with definite abnormality (table 1).

Table 1 EEG abnormalities in patients with bronchial carcinoma

\begin{tabular}{ll}
\hline Type of abnormality & $\begin{array}{l}\text { Frequency of } \\
\text { occurrence }\end{array}$ \\
\hline Slow posterior rhythmic & 3 \\
Excessive postcentral and temporal slow activity & 9 \\
(bilateral) & 2 \\
Generalised slow activity & 1 \\
Alpha asymmetry & 3 \\
Paroxysmal: & 1 \\
Focal sharp waves & 2 \\
Focal slow wave disturbances & 1 \\
Bilateral slow wave disturbances & 1 \\
\hline
\end{tabular}

Nerve conduction studies Although motor conduction velocity in the control nerves was marginally faster than in those of the patients the difference was not significant with the exception of the left median nerve. The mean motor conduction velocity for all the nerves taken together, however, was significantly slower in the patient group than in the controls (table 2). The amplitude of the evoked response to motor nerve stimulation was also significantly lower in the patient group than in the controls and the duration of the response significantly longer (table 3 ). The results of sensory conduction velocity measurement are shown in table 4. Although the velocities were within the normal range in nearly every nerve studied, conduction was faster in the control group than in the patients, and the amplitude of sensory nerve potentials was less in the patients than in the controls (table 3). A sensory potential was not obtained at the wrist in 20 sensory nerves in the patient group, compared with nine sensory nerves in the control group (by the Chi squared test this is significant $\mathrm{p}<0.05$ ). Although the means for motor and sensory nerve conduction velocity were within the normal range, a larger number of patients than controls had at least one nerve with a conduction velocity slower by more than two standard deviations below the control mean (table 5). The proportion of nerves in which this slowing occurred in patients and controls is shown in fig 1. Although the latency of the $\mathrm{H}$ reflex was longer in the patient group (33.1 ms, SD 3.33) than in the control

Table 250 patients with bronchial carcinoma

\begin{tabular}{|c|c|c|c|c|c|c|c|}
\hline \multirow[t]{3}{*}{ Nerve } & \multicolumn{7}{|c|}{ Motor conduction velocity $(\mathrm{m} / \mathrm{s})$} \\
\hline & \multicolumn{3}{|c|}{ Patients } & \multicolumn{3}{|c|}{ Controls } & \multirow[t]{2}{*}{$p^{*}$} \\
\hline & $n$ & Mean & $S D$ & $n$ & Mean & $S D$ & \\
\hline $\begin{array}{l}\text { Right common peroneal } \\
\text { Left common peroneal } \\
\text { Right ulnar } \\
\text { Left ulnar } \\
\text { Right median } \\
\text { Left median } \\
\text { Total of observations }\end{array}$ & $\begin{array}{r}48 \\
49 \\
50 \\
50 \\
50 \\
50 \\
297\end{array}$ & $\begin{array}{l}42 \cdot 9 \\
43 \cdot 8 \\
54 \cdot 1 \\
54 \cdot 0 \\
51 \cdot 9 \\
52 \cdot 8 \\
50 \cdot 0\end{array}$ & $\begin{array}{l}5 \cdot 24 \\
4 \cdot 93 \\
5 \cdot 60 \\
5 \cdot 59 \\
5 \cdot 66 \\
4 \cdot 57 \\
7 \cdot 03\end{array}$ & $\begin{array}{l}48 \\
49 \\
50 \\
50 \\
50 \\
50 \\
297\end{array}$ & $\begin{array}{l}42 \cdot 2 \\
45 \cdot 1 \\
54 \cdot 8 \\
55 \cdot 9 \\
52 \cdot 8 \\
54 \cdot 7 \\
51 \cdot 3\end{array}$ & $\begin{array}{l}4 \cdot 28 \\
4 \cdot 28 \\
5 \cdot 30 \\
5 \cdot 84 \\
4 \cdot 51 \\
4 \cdot 69 \\
6 \cdot 76\end{array}$ & $\begin{aligned} & N S \\
& N S \\
& N S \\
& N S \\
& N S \\
&<0.05 \\
&<0.01\end{aligned}$ \\
\hline
\end{tabular}

*Students paired $t$-test.

Table 350 patients with bronchial carcinoma

\begin{tabular}{|c|c|c|c|c|c|c|c|}
\hline \multirow[t]{3}{*}{ Nerve } & \multicolumn{7}{|c|}{ Sensory conduction velocity $(\mathrm{m} / \mathrm{s})$} \\
\hline & \multicolumn{3}{|c|}{ Patients } & \multicolumn{3}{|c|}{ Controls } & \multirow{2}{*}{$p^{*}$} \\
\hline & $n$ & Mean & $S D$ & $n$ & Mean & $S D$ & \\
\hline $\begin{array}{l}\text { Right ulnar finger to wrist } \\
\text { wrist to elbow } \\
\text { Left ulnar finger to wrist } \\
\text { wrist to elbow } \\
\text { Right median finger to wrist } \\
\text { wrist to elbow } \\
\text { Left median finger to wrist } \\
\text { wrist to elbow } \\
\text { Total of observations }\end{array}$ & $\begin{array}{r}45 \\
17 \\
42 \\
20 \\
45 \\
13 \\
34 \\
12 \\
228\end{array}$ & $\begin{array}{l}43 \cdot 3 \\
47 \cdot 8 \\
45 \cdot 1 \\
48 \cdot 4 \\
47 \cdot 5 \\
52 \cdot 2 \\
51 \cdot 2 \\
50 \cdot 0 \\
47 \cdot 3\end{array}$ & $\begin{array}{l}4 \cdot 78 \\
3 \cdot 84 \\
4 \cdot 97 \\
5 \cdot 76 \\
6 \cdot 59 \\
4 \cdot 80 \\
5 \cdot 41 \\
5 \cdot 95 \\
6 \cdot 06\end{array}$ & $\begin{array}{r}45 \\
17 \\
42 \\
20 \\
45 \\
13 \\
34 \\
12 \\
228\end{array}$ & $\begin{array}{l}45 \cdot 3 \\
53 \cdot 5 \\
47 \cdot 8 \\
54 \cdot 3 \\
52 \cdot 5 \\
52 \cdot 2 \\
52 \cdot 2 \\
57 \cdot 3 \\
50 \cdot 6\end{array}$ & $\begin{array}{l}3 \cdot 83 \\
5 \cdot 66 \\
4 \cdot 42 \\
7 \cdot 46 \\
6 \cdot 64 \\
5 \cdot 89 \\
6 \cdot 00 \\
5 \cdot 57 \\
6 \cdot 53\end{array}$ & $\begin{aligned} &< 0.02 \\
&<0.01 \\
&<0.025 \\
&<0.05 \\
&<0.001 \\
& \quad \text { NS } \\
& \quad \text { NS } \\
&<0.02 \\
&<0.001\end{aligned}$ \\
\hline
\end{tabular}

*Student's paired $t$-test. 
Table 450 patients with bronchial carcinoma. Amplitudes and duration of evoked muscle response (compound action potential) to nerve stimulation at wrist and ankle and amplitudes of sensory action at potentials at wrist and elbow

\begin{tabular}{|c|c|c|c|c|c|c|c|}
\hline & \multicolumn{3}{|c|}{ Patients } & \multicolumn{3}{|c|}{ Controls } & \multirow[t]{2}{*}{$p^{*}$} \\
\hline & $\bar{n}$ & Mean & $S D$ & $n$ & Mean & $S D$ & \\
\hline $\begin{array}{l}\text { Total motor response amplitudes } \\
\text { (compound action potential) }(\mathrm{mV})\end{array}$ & 237 & $8 \cdot 90$ & 4.93 & 237 & $10 \cdot 56$ & $5 \cdot 52$ & $<0.001$ \\
\hline $\begin{array}{l}\text { Total motor response durations } \\
\text { (compound action potential) }(\mathrm{m} / \mathrm{s})\end{array}$ & 193 & $11 \cdot 1$ & $1 \cdot 71$ & 193 & $10 \cdot 7$ & 1.67 & $<0.02$ \\
\hline $\begin{array}{l}\text { Total sensory potential amplitudes } \\
(\mu \mathrm{V})\end{array}$ & 187 & $6 \cdot 86$ & $2 \cdot 83$ & 187 & $7 \cdot 86$ & $3 \cdot 80$ & $<0.001$ \\
\hline
\end{tabular}

${ }^{*}$ Student's paired $t$ test.

Table 550 cases of bronchial carcinoma. Subjects with at least one conduction velocity more than $2 S D$ below control mean

\begin{tabular}{llll}
\hline & Patients & Controls & $p^{*}$ \\
\hline Motor & 13 & 5 & NS \\
Sensory & 14 & 2 & $<0.01$ \\
Motor and sensory & 20 & 7 & $<0.01$ \\
\hline
\end{tabular}

*Chi-squared test.

group (32.7 ms, SD 2.89), this difference was not statistically significant.

EMG findings In 27 of the patients no abnormality was recorded. In 13 spontaneous activity was seen, either as fasciculation potentials, positive sharp waves or fibrillation. Borderline abnormality was recorded in the remainder, in the form of an excess of insertion activity, of polyphasic or short duration potentials on voluntary contraction, or of a reduced interference pattern. Two patients had a motor unit pattern which was considered strongly suggestive of myopathy, in one case accompanied by signs of denervation.

Serum enzymes Five patients had a serum creatine kinase above the upper limit of normal

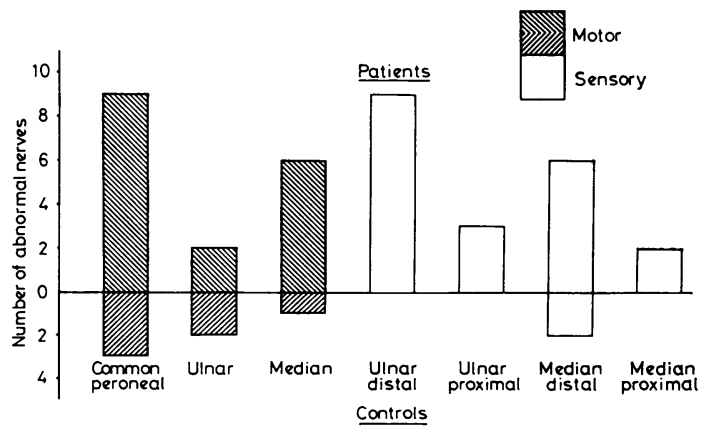

Fig 1 Histogram illustrating numbers of nerves in patient and control groups in which conduction velocit. was more than $2 S D$ below that of control mean.
(50 iu), the values ranging from $70-159 \mathrm{iu}$. Of these patients, four also had a moderate elevation of serum aldolase. A sixth patient had an elevated serum aldolase with a normal serum creatine kinase.

B Prospective study of bronchial carcinoma patients before and after treatment.

Studies prior to treatment

Clinical findings The majority of patients had predominantly respiratory symptoms, although one had a Pancoast tumour giving rise to weakness in the arm. On enquiry seven patients admitted to weight loss and generalised weakness, and one complained of selective weakness or cramps. Three had mild sensory symptoms in the hands. On examination three had absent vibration sense at the ankles, but there was no other evidence of sensory loss. Three patients had a mild degree of selective muscle weakness and wasting, involving the quadriceps in two and the thenar eminence in one. Ankle jerks were absent in two patients and occasional fasciculations were noted in various muscle groups in four patients. One patient had familial tremor and another exhibited early Parkinsonian features.

Nerve conduction studies Mean nerve conduction velocities for the patients with bronchial carcinoma came within the normal range. However, when the nerve conduction velocities in the patients were compared with those of the control population of 50 subjects previously studied, four patients were found to have slowing of conduction with velocities more than two standard deviations below the control mean in three or more nerves. Three patients had significant slowing of motor conduction in the legs and a further seven had significant slowing of sensory conduction in at least one nerve. Overall, $13 \%$ of the values obtained in the carcinoma patients were more than two standard deviations below the control mean. Applying Student's $t$ test, the mean sensory conduction velocity at the wrist was slower in the 
patient group than in the control population. That of the patients was $46.7 \pm 6.4 \mathrm{~m} / \mathrm{s}(\mathrm{n}=115)$, that of the controls $49.1 \pm 6 \cdot 2 \mathrm{~m} / \mathrm{s}(\mathrm{n}=187(\mathrm{p}<0.01)$. $E M G$ findings Spontaneous activity considered to represent fibrillation or positive sharp waves at two or more sites in a muscle was found in 12 patients. Fasciculation occurred in seven patients, in three in the absence of other spontaneous activity. In no patient was there a reduction in interference pattern and high amplitude so-called "giant units" were not seen. Spontaneous activity was commoner in distal muscles. In a single patient with weakness of one quadriceps muscle, polyphasic potentials were seen suggestive of a myopathy. In one patient a high frequency discharge occurred in the first dorsal interosseus muscle (figs 2 and 3).

Occasional spontaneous activity similar to that seen in the bronchial carcinoma patients were seen in four of the 20 controls $(20 \%)$, but no other abnormality was found. Some of the spontaneous activity seen was of low voltage (less than $20 \mu \mathrm{V}$ ) and in one of the four control subjects knee and ankle jerks were absent. Of 448 potentials examined in the right deltoid of the

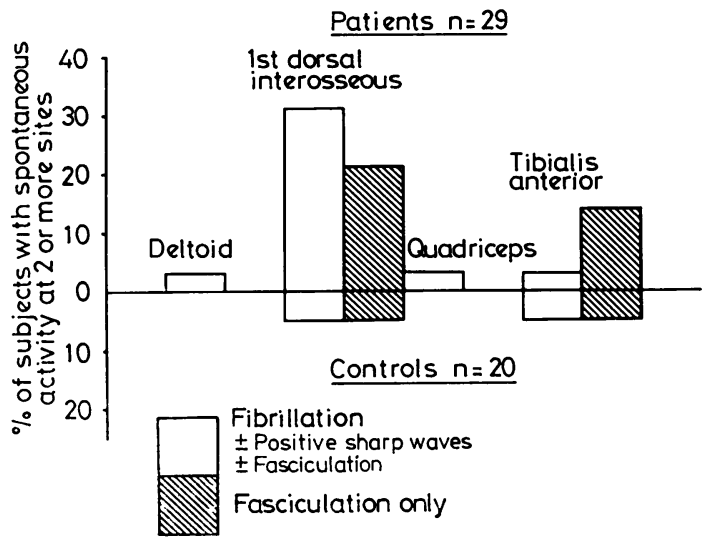

Fig 2 Distribution of spontaneous activity in patients and controls in prospective study. patients, 55 had five or more phases compared with 20 out of 352 potentials in the control group $(\mathrm{p}<0.005)$, Chi-squared test).

Repeat studies Nine patients treated for bronchial carcinoma returned for re-examination.

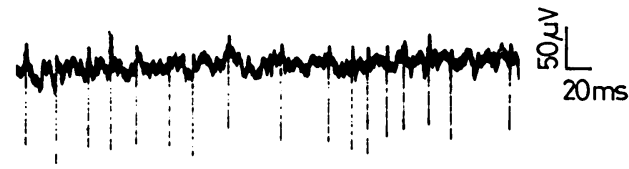

B
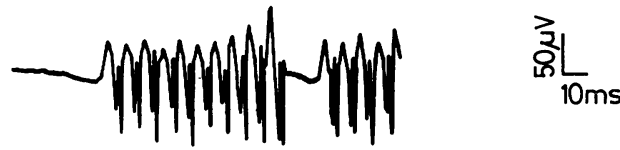

C

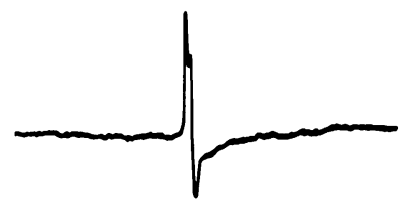

D

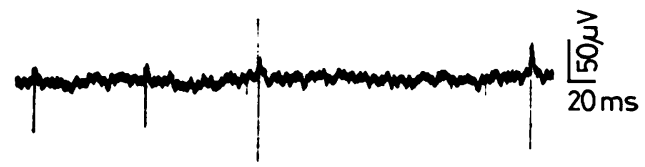

E

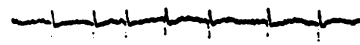

F
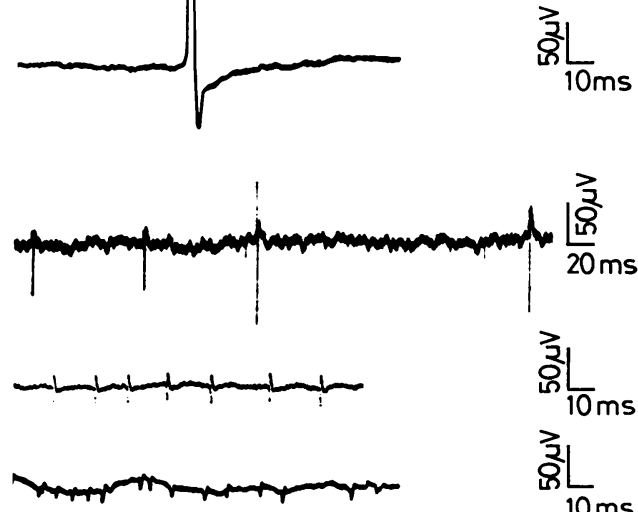

G
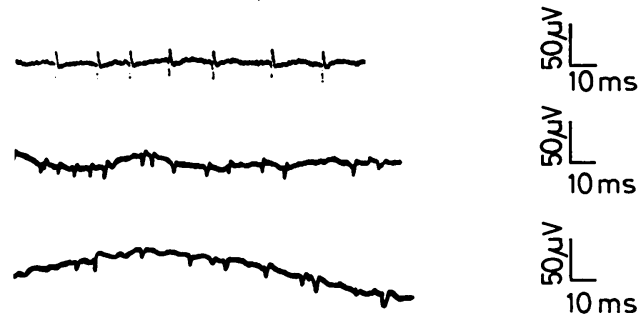

Fig 3 Examples of spontaneous activity. $A$ and $B$ represent repetitive discharges, $C$ fasciculation and $D, E, F$ and $G$ spontaneous diphasic potentials. All are from bronchial carcinoma patients with exception of $G$ which is from a control subject. Positive deflection downwards in every instance.

Table 6 Bronchial carcinoma. Conduction velocity and amplitude of potentials before and after treatment

\begin{tabular}{|c|c|c|c|c|c|c|c|}
\hline & \multicolumn{3}{|c|}{ Before } & \multicolumn{3}{|c|}{ After } & \multirow[t]{2}{*}{$p^{*}$} \\
\hline & $n$ & Mean & $S D$ & $n$ & Mean & $S D$ & \\
\hline $\begin{array}{l}\text { Motor conduction velocity }(\mathrm{m} / \mathrm{s}) \\
\text { Motor response amplitude } \\
\text { (compound action potential) }(\mathrm{mV})\end{array}$ & $\begin{array}{l}54 \\
41\end{array}$ & $\begin{array}{c}51 \cdot 5 \\
5 \cdot 63\end{array}$ & $\begin{array}{l}8 \cdot 71 \\
4 \cdot 14\end{array}$ & $\begin{array}{l}54 \\
41\end{array}$ & $\begin{array}{c}51 \cdot 1 \\
6 \cdot 27\end{array}$ & $\begin{array}{r}8 \cdot 20 \\
3 \cdot 84\end{array}$ & $\begin{array}{l}\text { NS } \\
\text { NS }\end{array}$ \\
\hline $\begin{array}{l}\text { Sensory conduction velocity } \\
\text { (finger to wrist) }(\mathrm{m} / \mathrm{s})\end{array}$ & 32 & $46 \cdot 8$ & 6.85 & 32 & $49 \cdot 9$ & $8 \cdot 80$ & $<0.05$ \\
\hline $\begin{array}{l}\text { Sensory potential amplitude } \\
\text { (at wrist) }(\mu \mathrm{V})\end{array}$ & 24 & $6 \cdot 14$ & $2 \cdot 39$ & 24 & $6 \cdot 36$ & $2 \cdot 29$ & NS \\
\hline
\end{tabular}

*Student's paired $t$ test. 
Eight had undergone successful surgical resection and one radiotherapy.

Clinical examination There was no significant change in the clinical neurological findings in any patient.

Nerve conduction velocity In seven out of nine of the patients mean conduction velocity was faster after treatment. Whereas motor conduction velocity was unchanged, there was a trend towards improvement in sensory conduction velocity $(p<0.05$. The small difference in amplitude of sensory potentials at the wrist before and after treatment was not statistically significant.

$E M G$ study No clear pattern of change in the EMG findings was evident. In two of the patients there was more evidence of spontaneous activity than before operation, but in three patients spontaneous activity found before operation was not evident on repeat examination (fig 4).

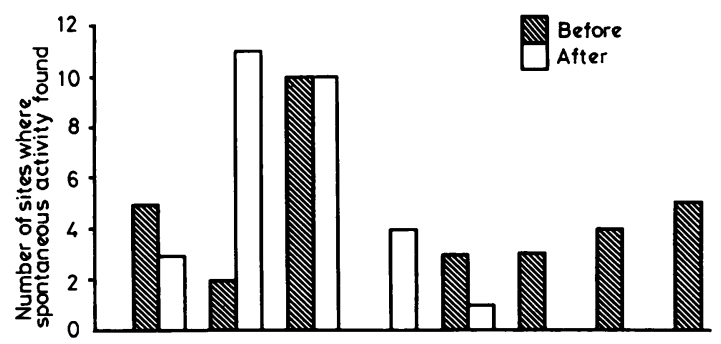

Fig 4 Histogram to illustrate spontaneous activity in eight patients with bronchial carcinoma before and after treatment. The ninth patient showed no spontaneous activity.

\section{Discussion}

Although minor neurological signs were seen in more than half the 80 patients with bronchial carcinoma, some of these probably were unrelated to the underlying carcinoma; they were also present in a number of control patients. Others such as hoarseness of voice, which in several instances was associated with vocal cord paralysis, may have been due to direct extension of the tumour; four patients who had focal neurological signs later developed evidence of cerebral metastases. No patient presented with a clinical picture of carcinomatous neuropathy, although in a few instances minor degrees of muscle wasting or peripheral sensory loss could represent the earliest signs of this disorder. This relatively low incidence of clinical abnormality contrasts with other findings in the literature ${ }^{38}$ and may be related to different patient selection or presentation of patients in the present study at an earlier stage of the disease.

The number of patients with bronchial carcinoma with EEG abnormalities is probably greater than in a healthy population of the same age, although it is difficult to obtain a fair comparison with a control population of hospital patients. The abnormalities recorded, however, many of which are non-specific, throw little light on the presence or absence of encephalopathy. In one patient who showed a paroxysmal abnormality in the EEG epilepsy subsequently developed and he was found to have an intracranial secondary deposit.

The nerve conduction studies in the 50 patients and in the matched controls are of interest in that although they show normal mean nerve conduction velocity in both patients and controls, there was a difference between the patient and control series, particularly striking for sensory conduction velocities. This was observed both when examining the means and also the individual records. Although marked slowing of conduction velocity did not occur, a significant number of patients had a degree of slowing greater than two standard deviations below the control mean. Other workers have not compared conduction velocity in bronchial carcinoma with a matched control series, but Campbell and Paty ${ }^{5}$ did find a number of patients in their series with mild degrees of slowing of nerve conduction velocity. The minor degree of slowing found in our study supports the conclusion of Trojaborg et $a l^{5}$ that the major change in peripheral nerves in patients with a bronchial carcinoma is one of axonal degeneration rather than segmental demyelination. The reduction in amplitude of the evoked muscle potentials is consistent with a fall-out of motor units, although the increase in duration of the potentials suggests that it could be accounted for in part by temporal dispersion. The presence of denervation changes on electromyography also is consistent with loss of motor units. The finding of an excess of polyphasic units in the patients in this prospective study is in agreement with the findings of Trojaborg et al, ${ }^{5}$ and is in favour of some degree of reinnervation having occurred at the time of investigation.

Although short duration or polyphasic potentials were seen quite frequently, in only three of the 79 patients who had electromyography was the appearance considered strongly suggestive of myopathy. The elevated serum creatine kinase recorded in five patients may be considered 
evidence of a myopathic condition. In only one of these was the EMG also suggestive of myopathy. This relatively small number of patients who showed myopathic features contrasts with the findings of Campbell and Paty, ${ }^{6}$ but is consistent with those of Trojaborg et al. ${ }^{5}$

In the pre-treatment assessment of patients with bronchial carcinoma only 14 of the 29 patients in whom an EMG was performed showed no evidence of spontaneous activity in the form of fibrillation, positive sharp waves or fasciculation. If these changes are accepted as evidence of partial denervation, the incidence of neuropathic change is found to be $52 \%$ which is consistent with previous studies. This is a higher figure than in the 50 patients who were matched with 50 controls; the explanation for this may be that, in the prospective study, abnormality was found most frequently in the first dorsal interosseus which was not examined in the first part of the present study in which only the proximal muscles were explored in every case. This preponderance of abnormality in distal muscles is consistent with the hypothesis that the principal change is one of axonal degeneration due to a "dying back" process.

Spontaneous activity also was evident in $20 \%$ of the controls, which is a higher proportion than is usually recognised. One of these controls had absent knee and ankle jerks, so it may be that the controls formed part of a hospital population that were not free of abnormality. Potentials which we have recognised as fibrillation have been diphasic or triphasic potentials with an initial positive deflection, a duration of less than $2 \mathrm{~ms}$ and an amplitude of $20-300 \mu \mathrm{V}$. In the end-plate zone fibrillation potentials have a negative initial phase and may be difficult to distinguish from end-plate noise or from diphasic potentials of negative onset which have been attributed to discharges from intramuscular nerve fibres. ${ }^{11}{ }^{12}$ Close to the endplate zone, fibrillation must be distinguished from diphasic potentials arising in the zone but seen at a distance. In the normal subjects, spontaneous activity was often accompanied by local discomfort. It is possible therefore, particularly in the distal muscles where the end-plate zone is relatively extensive, that some of this activity may represent action potentials arising in intramuscular nerve fibres.

The natural history of neuropathy in relation to bronchial carcinoma is unpredictable and Croft et $a l^{13}$ comment on the tendency to spontaneous improvement. Herishanu et $a \mathbf{l}^{14}$ found reduction in conduction velocity, particularly in patients who had undergone radiotherapy, and Croft and Wilkinson $^{8}$ suggested that treatment of the tumour might disturb the metabolic equilibrium of the nervous system. On the other hand, complete remission of a syndrome resembling motor neurone disease has been described after removal of a bronchial carcinoma. ${ }^{15}$ Longitudinal studies in this condition are difficult on account of the high mortality and significant degree of morbidity in the survivors. No more than 14 of our 30 patients ultimately underwent surgery, and only nine were re-examined six months later; no clear tendency to improvement was found. EMG findings were variable before and after treatment. It is difficult to attach much significance to the apparent trend towards an increase in sensory conduction velocity in such a small number, but it is consistent with our finding that the main abnormality in conduction velocity is in sensory nerves. Further prospective studies on patients with definite neurological deficits associated with tumour and who are amenable to surgical cure are necessary to clarify the effect of resection of a bronchial neoplasm on associated neuropathy.

We are grateful to $\operatorname{Dr} R \mathbf{N}$ Johnston, Dr $\mathrm{R} A$ Clark and Dr D H Smith for allowing access to patients under their care. Dr H H R Samarasinghe gave valuable assistance during the early part of this study and this is gratefully acknowledged. We are grateful to Mrs Catherine Kinnear, Mrs Ann Donald and Mrs Patricia Ireland for technical assistance.

\section{References}

1 Denny-Brown D. Primary sensory neuropathy with muscular changes associated with carcinoma. J Neurol, Neurosurg Psychiatry 1948; 11:73-87.

2 Brain R, Henson RA. Neurological syndromes associated with carcinoma. The carcinomatous neuromyopathies. Lancet 1958; ii:971-4.

3 Croft $\mathrm{PB}$, Wilkinson $\mathrm{M}$. The course and prognosis in some types of carcinomatous neuromyopathy. Brain 1965; 88:427-34.

4 Moody JF. Electrophysiological investigation into neurological complication of carcinoma. Brain 1965; 88:1023-36.

5 Trojaborg W, Frantzen E, Andersen I. Peripheral neuropathy and myopathy associated with carcinoma of the lung. Brain 1969; 92:71-82.

6 Campbell MJ, Paty DW. Carcinomatous neuromyopathy: 1. Electrophysiological studies. An electrophysiological and immunological study of patients with carcinoma of the lung. J Neurol, Neurosurg Psychiatry 1974; 37:131-41.

7 Paul T, Katiyar BC, Misra S, Pant GC. Carcinomatous neuromuscular syndromes. A clinical and 
quantitative electrophysiological study. Brain 1978; 101:53-63.

8 Croft PB, Wilkinson M. Carcinomatous neuromyopathy. Its incidence in patients with carcinoma of the lung and carcinoma of the breast. Lancet 1963; 1:184-8.

9 Croft PB, Wilkinson M. The course and prognosis in some types of carcinomatous neuromyopathy. Brain 1969; 92:1-8.

10 Lenman JAR, Ritchie AE. Clinical Electromyography, 2nd Edn, 1977. Pitman, London.

11 Jones RV, Lambert EH, Sayre GP. Source of a type of "insertion activity" in electromyography with evaluation of a histologic method of localization. Arch Phys Med 1955; 36:301-10.
12 Buchthal F, Rosenfalck P. Spontaneous electrical activity of human muscle. Electroencephalog Clin Neurophysiol 1966; 20:321-36.

13 Croft PB, Urich $H$, Wilkinson M. Peripheral neuropathy of sensorimotor type associated with malignant disease. Brain 1967; 90:31-66.

14 Herishanu Y, Wolf E, Taustein I, Biran S. The carcinomatous neuropathy. A clinical and electrophysiological comparative study, with special reference to the effect of treatment on the nervous system. Euro Neurol 1970; 4:370-4.

15 Mitchell DM, Olczak SA. Remission of a syndrome indistinguishable from motor neurone disease after resection for bronchial carcinoma. Br Med J 1979; 2:176-7. 